In this paper, we showed the experience, application and effect of various treatment models, including telepsychiatry use for psychometric instruments, tests and scales. Clients' examinees have given their consent to participate in the research and treatment of mobbing phenomena.

Participants, Materials/Methods: Total sample data is 220 examinees, in the age of 18-65 years, of both sexes, all working people, of different social status and cultural habits. They have been treated in Center for telepsychiatry in virtual psychiatry Ambulance for various psychiatric syndromes all having the same denominator-mobbing, as ethiopathogenetic factor. In the research, the following instruments were used: Questionnaire of socio-demographical, Telepsychiatric interview (with teleconsultation), Beck's scale for self-esteem of depression, Hamilton's scale for depression (HAM-D) and Hamilton's scale for anxiety (HAM-A).

Results: Upon evaluation, it was identified that major proportion of examinees shows:

1) Mental health and psychiatric diagnosis $142(64,55 \%)$

2) And other Different somatic diagnosis 74 (33, 64\%)

$3)$ And only $4(1,81 \%)$ without any diagnosis.

4) From $142(64,55 \%)$ Mental health and psychiatric diagnosis major $110(77,46 \%)$ include symptoms of depression; that is statistically important $P<0.01$.

Conclusions: With treatment by telepsychiatry with teleconsultation result is significantly reduces consequences to mental health. These facts are best confirmed by measurement instruments used during this research: HAM-D, Beck's scale and ICD 10. Phenomena of abuse on workplace should be continuously researched, and all gained results could have significant clinical, public health and research implications.

62

Untreated schizophrenics in hard felony commitment Tanja Mijatovic-Papic, Nevenka Duletic \& Aleksandar Tomcuk Forensic ward, Psychiatric Hospital Kotor Dobrota b.b.. 85330 Kotor, Montenegro

E-mail: spbdobrota@cg.yu

Introduction/Objectives: Analysis of diagnostic categories in respect to character of felony committed, among the patients on forensic ward of Specialized Psychiatric Hospital Kotor, Montenegro, reveals that 5 of 21 hospitalized patients who committed homicide where tempore criminis in a state of unaccountability due to mental illness - paranoid schizophrenia psychosis.

Participants, Materials/Methods: In 4 of 5 cases mentioned, illness was diagnosed for the first time during forensic psychiatric expertise and so, based on assessment of clinical status of subject and their occupational and social disfunctionality, it is ascertained that the beginning of illness dates from the period much prior to the crime committed, but the patients didn't have psychiatric treatment and have never taken antipsychotic therapy.

Results: Regular application of psychopharmacological protocol led to a reduction of psychotic phenomenology and, according to indicators on scale of aggression, to a significant decrease in estimated risk from auto and hetero destructive behavior.

Conclusions: Regarding that further larger number of chronically ill patients who suffer from paranoid schizophrenia and are regularly treated never committed a crime in their personal history (do not have criminal behavior in their personal history), even though it is a psychiatric disorder permanent in character, mentally ill patients cannot be considered permanently aggressive toward the surroundings or themselves.
63

\section{Psychosis and Parkinson's disease}

Tomislav Peharda, Eva Zorijan, Ivica Šain \& Dragutin Breški

Psychiatry, OB Pula Zagrebačka 30, 52100 Pula, Croatia

E-mail: evazorijan@yahoo.com

Introduction/Objectives: Psychosis in Parkinson's disease can be caused by both extrinsic and intrinsic factors.

Participants, Materials/Methods: Case presentation: In the period from 1982 to 1986 the patient S.A. (75-year-old woman) had been hospitalized several times. She was admitted to the psychiatry with the diagnosis psychosis depressive and cured with antipsychotics (clozapine, levomepromazin) and antidepressants (maprotilin). In 2009 she was again admitted, but this time in the semistuporous state, mutistic, with the clinical state of paranoid - halucinatory psychosis. She was diagnosed Parkinson's disease 2 years ago and has been treated with the combination of levodopa and benzerazid as well as entekapon.

Results: The question is whether the trigger for the psychosis is Parkinson's disease, antiparkinsonian medications in itself, or the previous psychiatric diagnosis represents the risk factor which enables the antiparkinsonian medications to activate the psychosis. The treatment involved gradual decrease in the dosage of antiparkinsonian medications and introduction of antipsychotics (clozapine).

\section{4 \\ Benzodiazepine derivatives consumption in the Republic of Croatia in 2005, 2006 and 2007 \\ P. Draganic, S. Zezelic, V. Macolic-Sarinic \& S. Tomic Medical department, Agency for medicinal products and medical devices Ksaverska cesta 4, 10000 Zagreb, Croatia \\ E-mail: pero.draganic@almp.hr}

Introduction/Objectives: Benzodiazepine derivatives are drugs used to relieve anxiety, muscle spasms, and seizures and to control agitation caused by alcohol withdrawal. A review of the cost and consumption for all medicines and benzodiazepine derivatives in Croatia in 2005-2007 periods, as well as the data on medicine usage for these two groups are obtained. The importance of collecting data on benzodiazepine derivatives consumption is in correlation with the improvement on antipsychotic/anxiolytic therapy.

Participants, Materials/Methods: Agency for Medicinal Products and Medical Devices in Croatia is authorized for collecting and processing data on medicines consumption. Data on medicine usage between 2005 and 2007 was gathered from obliged entities, processed by the DDD/1000inh/day and ATC classification, and the analysis of the financial indicators was done as well.

Results: It was established that the total medicines market in Croatia in 2005 was $€ 520$ million and $8.5 \%$ DDD/1000inh/day for benzodiazepine derivatives N05BA + N05CD groups, in 2006 it was $€ 562$ million and $8.53 \% \mathrm{DDD} / 1000 \mathrm{inh} /$ day for N05BA + N05CD groups, and in 2007 it was $€ 670$ million and $9.37 \%$ DDD/1000inh/ day for N05BA + N05CD groups. By processing consumption data as DDD/1000inh/day, between 2005 and 2007 consumption of $\mathrm{N} 05 \mathrm{BA}+\mathrm{N} 05 \mathrm{CD}$ groups were 55.15, 59.37 and $76.5 \mathrm{DDD} /$ $1000 \mathrm{inh} /$ day, respectively. Expenditure of benzodiazepine derivatives individually was as follows: diazepam was at the first place (20.71 in 2005, 23.02 in 2006 and $23.42 \mathrm{DDD} / 1000 \mathrm{inh} /$ day in 2007. Alprazolam was at the second place (13.99 in 2005, 16.14 in 2006 and 16.9 DDD/1000inh/day in 2007.

Conclusions: During the period 2005-2007, all benzodiazepine derivatives, which were in the market in Croatia, showed a continuous increase in prescribed DDD/1000inh/day. For the entire period diazepam and alprazolam were the most prescribed 